\title{
The Rhode Island Dialect:
}

\section{An Investigation of Language Change in New England}

\author{
By Moira Crocker
}

\section{Introduction}

As evidenced in the 2010 article from American Speech, "Farewell to the Founders", dialect boundaries in New England are not only shifting eastward, but the use of traditional phonological New England features is becoming significantly reduced in the speech of younger generations of speakers (Stanford, Leddy-Cecere \& Baclawski). This was determined using a study that looked at both real-time and apparent-time results to determine the use of regional phonological features amongst speakers along the historic East-West New England dialect boundary as well as comparing the speech of younger and older generations. The features that are most rapidly disappearing from the younger generation's speech were those that are considered stigmatized, reflecting the influence of social change on the status of New England dialects.

Rhode Island speech, as a whole, is largely stigmatized, and regional features include non-rhotic speech, a feature which has had consistently negative perceptions by those both within the speech community and without. In order to assess if any similar such reduction in the Rhode Island dialect is taking place, I investigated the speech patterns and language attitudes of speakers both through an online survey as well as through brief linguistic interviews. I targeted both phonological and lexical features when determining informants' use of the Rhode Island dialect in order to get a fuller understanding as to what categories of features were undergoing change between generations. I hypothesized that, in keeping with the pattern of eastern-shifting isoglosses over time in other areas of New England, the features of the Rhode Island dialect are similarly receding in the speech of the younger generations in the state. 


\section{Methodology}

This study was an examination of the speech of native or near-native Rhode Islanders to determine whether, like other regional dialects of the area, the features of the dialect are diminishing over time. To do so, I collected data on the lexical and phonological properties of the speech of members of the younger generation and compared it to that of older speakers. In addition to retrieving data on the speech usage of these informants, I also looked at their attitudes towards the dialect and its features to help determine the influence of social factors on the region's language use.

\subsection{Subjects}

Due to the lack of prior research conducted on the Rhode Island dialect, I created an apparent-time study contrasting the language use of speakers of three different age groups: those twenty-five and under, those who were between twenty-six and forty-five, and those over forty-five. The oldest and youngest groups served as my target demographics while those in the intermediate age group functioned as a control, as well as providing meaningful data about the patterns and rate of the apparent change. All participants were native or near native to Rhode Island, meaning that they have lived in the state since early childhood.

191 total participants took the online survey, of which 163 were native to Rhode Island. The remaining participants from out of state were excluded from the remainder of the survey by a logic function within the program. Of the informants who qualified, $24 \%$ were male and $76 \%$ female with $47 \%$ of respondents falling into the youngest generation, $20 \%$ in the intermediate, and $33 \%$ in the oldest. $92 \%$ of the respondents identified as white, $4 \%$ as African American, $1 \%$ as Asian American, $4 \%$ as Latino or Latina, $1 \%$ as Middle Eastern, and an additional $1 \%$ as Native American. This racial composition of informants mirrors that of the state as a whole (United States Census Bureau), as shown in Figure 1 below. Of the thirty-nine towns in the state, all but Smithfield were represented in the survey. 


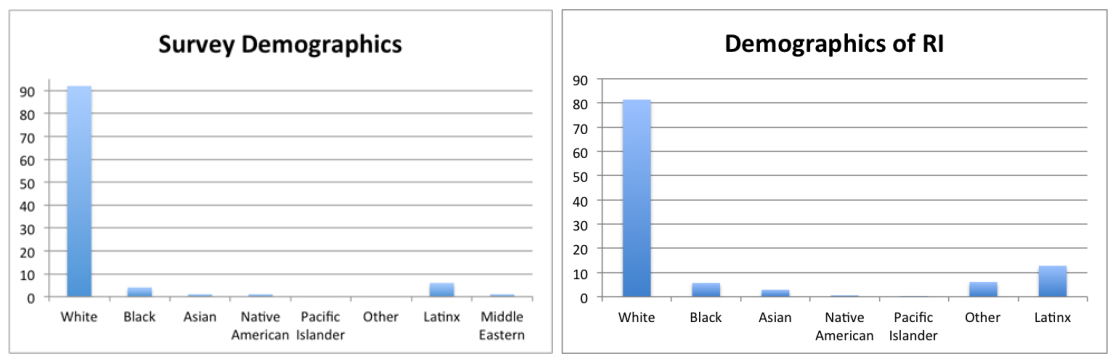

Figure 1: Demographics of state versus survey participants

Interviews were conducted with thirteen speakers native to Rhode Island, divided into four cells: Three female participants forty-six and over, two male participants forty-six and over, four female participants twenty-five or younger and four male participants twenty-five and younger. Of the two groups representing the older generations, all speakers were white while both younger groups were composed of two white speakers, one Latino and Latina respectively, and one African American. Neither the survey nor the interview controlled for socio-economic class or education. The influence of languages other than English was also accounted for, although a number of participants underreported their fluency, perhaps due to the power dynamics and awareness of languages such as AAVE (only one respondent claimed AAVE despite a number of speakers exhibiting AAVE in their interviews and some in their surveys as well). One speaker also mentioned in his interview that he grew up speaking French but he did not list that as a language he controlled natively or with native-like fluency. This could be related to the phrasing of the question, since heritage speakers often do not feel as though they have complete control over the language's grammar, although it does still hold influence over their speech. This also led me to believe that other speakers could have similarly omitted languages of influence, and as a result, I did not examine the influence of this factor closely in this study.

\subsection{Materials}

For this study, I created a twenty-five-question survey using Qualtrics software (Appendix 8). The first six questions asked demographic information, 7-20 examined personal language use with regard to lexical items and phonology, and questions 21-25 asked about respondents' perceptions of the dialect and their own language use. These questions were selected to reflect some notable features of the dialect, although there is an emphasis on lexical items due to the nature of taking a survey online since it relies on self-reporting. Such features included non-rhoticism and a vowel 
distinction commonly referred to as the Mary/merry/marry distinction, as well as some of the typically distinctive regional lexical features, such as "quahog" for "clam" and "bubbler" for "water fountain".

I also used a script of my own design (Appendix 1) for the interview portion of the study in order to create the opportunity for regional phonological features to be elicited. This script particularly created the context necessary for the use of features such as non-rhoticism, the lowering and fronting of [o] to [a] such as in the word "Florida", the Mary/merry/marry distinction, as well as r-insertion. I also included a number of terms specific to Rhode Island in an attempt to further elicit the dialect, since these are the terms that are most likely to exhibit regional features because most of speakers' input has been produced by other native Rhode Islanders. The script was also written informally and in a manner reflective of natural speech in order to attain the most natural speech possible from a written script.

I used my laptop to record the entirety of the interviews, with the hope that a laptop would bring less attention to the fact that speakers were being recorded than another device to best avoid the observer's paradox. Since their speech was being recorded, I also used an informed consent form (Appendix 2) to provide informants with the necessary information regarding their participation, as well as to legally be able to record their speech.

\subsection{Procedure}

I used the snowball effect to spread my survey, sharing it on social mediaboth Facebook and Twitter - and asking others to share it with people they knew as well. The survey was available for just over two weeks due to time constraints. For the second portion of my study, I gathered participants whom I either knew personally or met through a mutual friend and had spent time with prior to the interview in order to try to get speakers comfortable to use their natural speech around me. The interview itself had a three-part process: (1) informants would first complete the survey (but on paper rather than online so I could compare their responses to the data from the interview). (2) I would then ask them to read the passage aloud, and finally (3) we would finish the interview with a fairly open conversation wherein I asked them their impressions about the Rhode Island dialect, as well as who in the state was likely or not likely to use it. I then not only compared their scripted speech to their free speech, but also their use of phonological and even lexical features to their survey responses. 


\section{Results and Discussion}

\subsection{Survey}

\subsubsection{Phonological Features}

There was a stark contrast in the results of the survey regarding the use of regional phonological features between the different generations. There were two different regional variations examined in this survey: the Mary/merry/marry distinction and non-rhotic speech. Although this study is reliant on self-reporting which can be unreliable, particularly with stigmatized features, a comparison between the survey results and the recorded speech of informants who participated in both aspects of the study indicates that participants correctly self-reported the features of their own speech.

The first feature, the Mary/merry/marry distinction was present in the speech of $67 \%$ of the oldest generation, $57 \%$ of the intermediate generation, and just $26 \%$ of the youngest group This shows a remarkable decrease of $41 \%$ between the oldest and youngest informants, with the control group showing a slight decrease from the oldest generation. This significant difference in the rate of use amongst speakers of different age groups suggests a decline in the feature over time.

The second phonological feature, non-rhotic speech, is perhaps the most salient feature examined in this study. Non-rhoticity is one of the features that is most heavily associated with New England speech, and one of the most stigmatized. This perception is not lost on native Rhode Islanders, with the majority of speakers describing Rhode Island speech primarily based on its lack of rhoticity and sometimes r-insertion, with one speaker describing the dialect as "lots of r's making guest appearances, and lots of r's on vacation." $84 \%$ of speakers under twenty-five reported rhotic speech, as well as $67 \%$ of the control group and $49 \%$ of the oldest group. This shows a $33 \%$ decrease between the two target groups, with the control group again exhibiting the feature at an intermediate rate. This not only reflects a decrease in the pervasiveness of the feature over time, but that it is diminishing at a similar rate as the Mary/merry/marry distinction as seen in Figure 5 below. That the feature is decreasing at a similar rate reflects that, although speakers have a greater linguistic awareness of the social connotations of non-rhotic speech, both phonological features of the dialect 
are similarly decreasing, although non-rhotic speech is used at a lower rate overall.
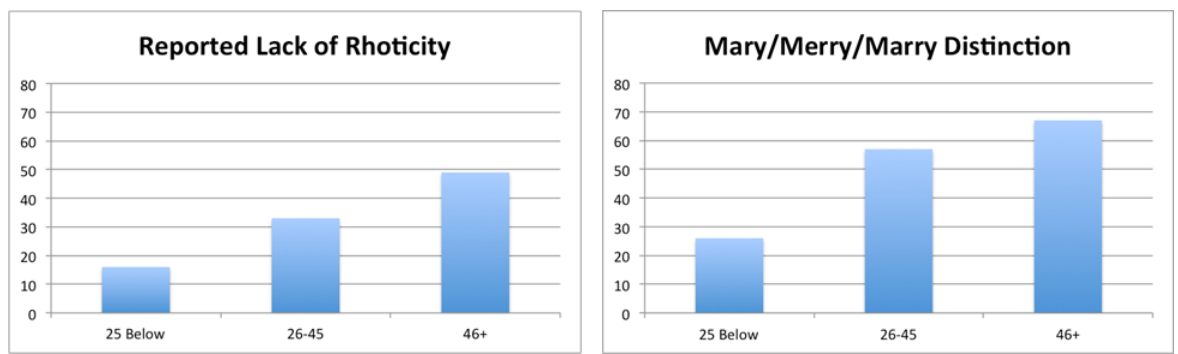

Figure 5: Comparison of Phonological Results

\subsubsection{Lexical Features}

The results for lexical features of the dialect were much more varied than the use of phonological features inter-generationally. The patterns of lexicalfeature usage can be divided into three major groups: features with a significant decreased used amongst the younger generation as compared to the oldest participants, features with no significant change intergenerationally, and features which are emerging or experiencing increased use amongst younger informants.

Lexical items that decreased significantly include 'quahog', 'elastic', 'jimmies', and 'cabinet'. These terms were of varying overall usage, with more than half of younger speakers continuing to use 'quahog' and 'jimmies', whereas the usage of both 'elastic' and 'cabinet' was below $20 \%$. 'Package store' also most fits this group, because of the significant decrease between the oldest and youngest generation from $39 \%$ to just $18 \%$.

However, this term's distribution is something of an anomaly due to the jump in usage by the intermediate age group, in which $60 \%$ of speakers ages twenty-six to forty-five used the regional term as compared to the other two groups usages, which were $21 \%$ and $42 \%$ lower. This pattern shows an overall decrease in the use of some lexical features of the dialect corresponding to age, resulting in a drop in the use of both features that are relatively pervasive amongst the oldest generation and those with a much lower frequency as well.

The cases of 'rotary' 'clicker' and 'bubbler' show the persistence of some lexical features over time, since all of these terms retain high usage with minimal change in use over generation, although 'rotary' does show more 
consistent change over time than 'clicker' or 'bubbler'. 'Bubbler' is a particularly pervasive lexical item, with over $80 \%$ of speakers from each age group listing it as their primary lexical item for the object in question. The consistency in the usage rates of these terms show that any decrease that is occurring in the dialect between generations is not happening evenly on the lexical level, but rather the use of some terms is diminishing rapidly while other terms are experiencing no significant rate of change.

Perhaps one of the most interesting lexical results of the survey is the emergence of a new lexical item amongst the younger two generations as a reference for milkshake. While 'cabinet' is the typical regional term for a milkshake, $7 \%$ of the control group and $11 \%$ of the youngest informants identified the stimulus as 'Awful Awfuls', while no one from the oldest age group used this term, as shown in Figure 4 below.

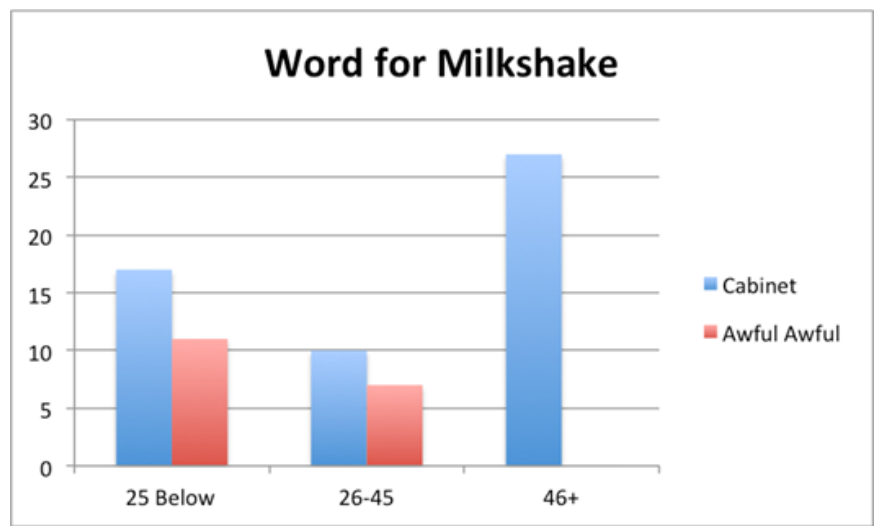

Figure 4: Results of Use of Terms for Milkshake

These rates are comparable to the usage of 'cabinet' for the youngest two generations, which were $17 \%$ and $10 \%$ for the youngest and intermediate groups respectively. The pattern of 'cabinet' shows a decrease between the oldest and younger generations, but the emergence of the new feature starting with the control group gains momentum in use amongst the youngest generation of Rhode Islanders. This term is derived from a local restaurant chain's term for their own milkshakes, which has undergone generification and has become an acceptable term for all milkshakes at an increasing rate between the younger two age groups. The image used supports the notion that this is in fact a new lexical item for milkshakes in general, since the image depicted generic milkshakes not reflective of the brand that originated use of the term. 
Another lexical item that is increasing amongst the younger generations is the term 'wicked'. Only $68 \%$ of the oldest generation claimed to use this term at least sometimes, compared $90 \%$ of the control group and $89 \%$ of the youngest group. This again reflects the uneven nature of the recession of the regional dialect amongst younger speakers, not only in that some terms are actually increasing, but in that a considerable number of lexical features are not being reduced gradually throughout generations. In some cases, the control group's speech more closely resembles the patterns of the youngest group (such as in the case of 'wicked') while in other cases, uses of regional features at either a comparable or even higher rate than that of the oldest generation, a pattern that is not necessarily expected nor reflected in the case of phonological features.

\subsubsection{Speech Perceptions}

Regarding speaker's perceptions of the dialect, qualitative responses showed a fairly even distribution of overtly positive, negative, and neutral responses between the three age groups, with $24 \%$ responses being positive, $32 \%$ negative, and $44 \%$ neither. Common positive responses included "I love it", "It reminds me of home" and "it's lit fam", while the vast majority of negative responses said the dialect sounded "uneducated". In the quantitative results using ten-point-scales of correctness and pleasantness, the oldest generation scored the dialect lowest for both pleasantness and correctness, rating it an average of 5.23 for correctness and 5.73 for pleasantness. The control group rated it highest for pleasantness, with an average score of 6.37 while the youngest group rated it highest for correctness at 6.24 .

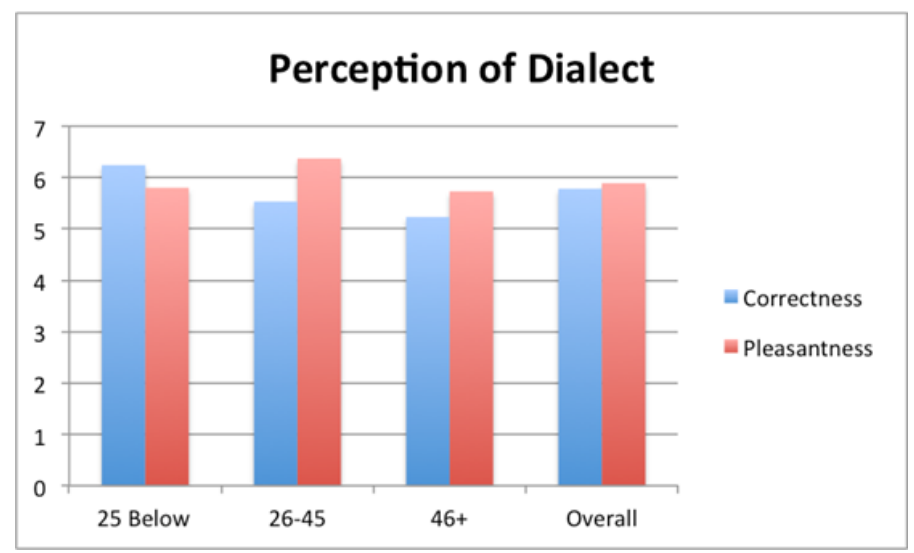

Figure 2: Quantitative Perceptions Results 
While there was not a tremendous difference in perceptions of correctness and pleasantness in between age groups, the oldest generation clearly shows the harshest perceptions of the dialect. When asked about their own speech

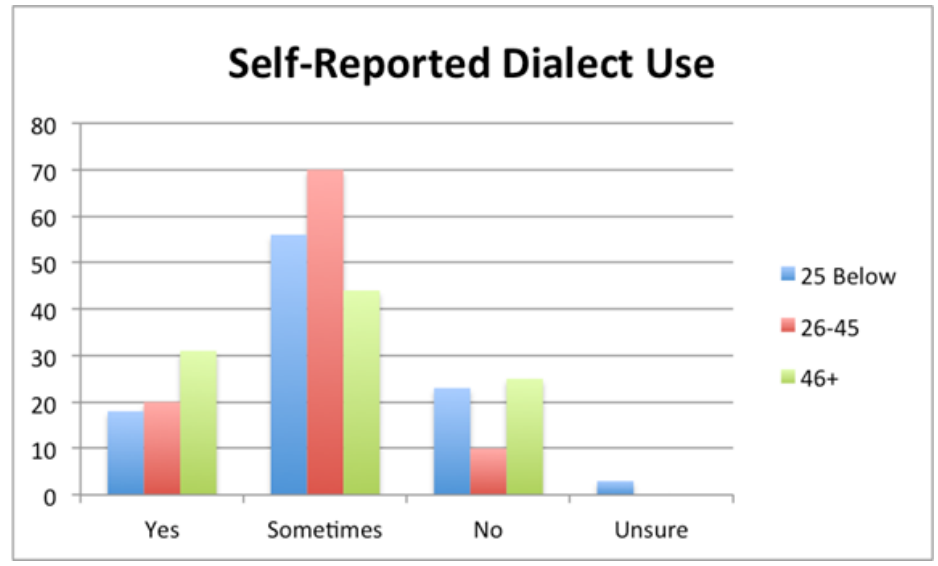

Figure 3: Self-Reported Dialect Use

and if they used the dialect themselves, the oldest group had the highest percentage of speakers who denied using it despite having the highest rate of use of regional features out of the three age groups. This could be due to the group's more negative perception of the dialect, since this group rated it lower in both correctness and pleasantness than the other two groups. In the qualitative response section, many of the older speakers noted that the dialect sounded "uneducated", and likely want to distance themselves from the dialect's stigma. It is also possible that these speakers are simply less aware of their own language use, or of the features of the dialect itself. Both younger groups were more likely to say they only used it sometimes or were unsure as apparent in Figure 3 below, which could be due to their continued use of some lexical items but not phonological items

\subsection{Interviews}

\subsubsection{Phonological Features}

The interview results regarding phonological features mirror the results of the survey both in the recorded and free speech of the informants. All of the male speakers from the oldest group exhibited both non-rhoticity and vowel changes characteristic of the dialect, including the Mary/merry/marry distinction as well as the lowering of [o] to [a], like in the word 'Florida'. The female informants from this age group exhibited target phonological features at lower rates than their male counterparts, with only one of the 
three exhibiting non-rhotic speech; and, while all used at least some instances of vowel change in both free and scripted speech, they did not do so consistently. This could be affected by their career paths, as both were teachers and one mentioned that in order to get her job within the state, she had to take a class on pronunciation and was told she must "use her r's". This prescriptive push back against phonological aspects of the languageparticularly non-rhotic speech-could help explain the consistent decrease in the use of its phonological features inter-generationally. Nearly all speakers reported experiences with prescriptive grammar, particularly with being told to avoid non-rhotic speech. This pushback, which focuses specifically on phonological features, could contribute to the apparent decrease in the dialect's use amongst the younger generation.

The younger speakers exhibited very few phonological features of the Rhode Island dialect. Only one female informant from the younger group used a vowel shift of the dialect, the lowering of [o] to [a] in the word 'Florida' in the elicited speech, which she immediately self-corrected. There were three instances of younger speakers using non-rhotic speech, however they were all also fluent speakers of AAVE_-although not all of them self-reported this fact-and since they did not also exhibit r-insertion in their speech, it is more likely that this feature correlates to their use of AAVE rather than the regional dialect.

\subsubsection{Speech Perceptions}

As far as perceptions about speech, all informants noted a definite connection between the dialect and where in the state speakers were from and their age. Some speakers also connected ethnicity to use of the dialect, although not all who did could specify which ethnicities were more or less likely to use it. Nearly every speaker said the towns of Cranston, North Providence, Johnston and Federal Hill were more likely to use the dialect, with many saying each of these towns had its own variant of the dialect. Cranston was particularly criticized, with many speakers both in the survey and the interview said their speech was very unpleasant; one speaker saying he scored the dialect as a five for correctness, only because of "how bad people from Cranston speak". Six out of the eight younger speakers said that "only white people" used the dialect, with many from all age groups noting that Italian-Americans in particular were more likely to use the dialect. Every single informant said that older speakers were much more likely to use the dialect than those of younger generations. 
Participants also seemed to have a hard time identifying the specific features of the dialect. All speakers immediately listed non-rhotic speech when asked about the dialect, but many could not name any features beyond that.

However, one speaker offered to read the script in her best impersonation of the dialect and exhibited a number of vowel changes, showing an awareness of other features although she only listed "r-lessness" when describing the sound properties.

The younger generation also showed a greater metalinguistic awareness than the older group. For example, one of the older males said that he did not use the dialect, although he exhibited all the phonological features as well as a number of lexical items. It's also important to note that he didn't deny using the features; he just denied that he used the dialect. Younger speakers however were able to note that while they did not use phonological features, they did use some of the lexical items, some of which they felt passionately about. 'Bubbler' was the most common term whose use younger speakers were adamant about, with one speaker mentioning his experience with students at his college not from Rhode Island calling it a water fountain saying, "no-it's the bubbler".

\section{Conclusion}

This overall pattern of decrease in the use of regional features indicates a reduction in the dialect with younger generations-though not in its entirety. Features that speakers more readily associated with the region and the pejorative connotations of its dialect, such as non-rhotic speech, decreased consistently between generations in the speech of participants. However, other features are being retained in the speech of native Rhode Islanders, particularly certain lexical items, such as "bubbler", "rotary", and "clicker", and there is even the emergence of new regional terms, as seen in the use of "Awful Awful" for milkshake in younger participants' speech. The reduction of phonological features of the dialect but retention of its lexical terms reflects a decrease in the aspects of the dialect that are more closely associated with the pejorative views of the region's speakers, while other less stigmatized features are being retained. This supports my hypothesis to a degree, as there is a clear distinction between the language use of different generations, however this study points more to a shift in the dialect's features rather than its decline over time. 


\section{Future Study}

While the data appears to support my hypothesis to a degree, it is important to note that due to the lack of prior research on language use in Rhode Island, it is impossible to tell whether these patterns of dialect use between generations is the result of language change or age-grading. Further research will be needed to determine this, as well as to address more thoroughly the phonological aspects to determine if they are in fact receding consistently, or if as a whole there are sub-patterns as seen the in usage of lexical features.

Additionally, further research that more closely examined how other factors, such as socio-economic class and education, play into the use of the dialect could prove informative, particularly since so many speakers felt that the dialect sounded "uneducated".

Several changes to the study could have improved it and rendered clearer results. The first would be to include more phonological characteristics to get a fuller understanding of the patterns of dialect change. Interviewing a greater number of participants would also provide better results, as would asking speakers to list their specific age rather than having such a large control group, particularly since the control group's responses tended to be less consistent in its trends than the other two. Further research addressing the isogloss of 'Awful Awful' as well as determining if any other lexical features are emerging could help provide definitive conclusions about the state and manner of the dialect change within the state. 


\section{Works Cited}

"Rhode Island QuickFacts." United States Census Bureau, April 2015, https://www.census.gov/quickfacts/table/PST045215/44/accessib le.

Stanford, James N., Thomas A. Leddy-Cecere, and Kenneth P. Baclawski. "Farewell to the founders: major dialect changes along the EastWest New England border." American Speech 87.2 (2012): 126169. 


\section{Appendices}

\section{Appendix 1: Script}

Last year my aunt Mary drove her car up from Florida to visit my father and the rest of their family. She has a daughter who is kind of weird and doesn't normally talk much. Our family is huge since my father has four brothers and a sister, but they don't always think it's a good idea to see each other. Most of them live in Rhode Island, in Cranston, North Providence, and Narragansett down by the water. Most of them don't have animals, but my uncle Peter has a huge dog.

\section{Appendix 2: Informed consent form}

\section{Informed Consent Form}

$\mathrm{I}$, use and attitudes in Rhode Island. , agree to take part in a study of language

I understand that my speech will be recorded both as I recite a passage and while I participate in a conversation regarding language use in Rhode Island.

I understand that all personal information will be confidential and that my answers will remain anonymous.

I understand that I am under no obligation to participate in this study.

I understand what this study involves and agree to participate.

Signature

Date

\section{Appendix 3: Qualtrics Survey}

[Available as a supplementary file] 